series analyses. (Huang W-T, Gargiullo PM, Broder KR, et al. Pediatrics August 2010;126:e263-e269). By contrast published studies report a relative risk of 3.3 for febrile seizures within 0-3 days of DTP whole-cell pertussis vaccine (RR 5.7 on the same day as vaccination). (Barlow WE et al. New Engl J Med 2001;345:656-661).

\title{
YIELD OF LUMBAR PUNCTURE WITH FIRST COMPLEX FEBRILE SEIZURE
}

Researchers in emergency medicine and pediatric neurology at Children's Hospital Boston, MA, assessed the rate of acute bacterial meningitis (ABM) among 526 children (56\% male) aged 6 to 60 months (median age 17 months) who were evaluated in the ED between 1995 and 2008 for a first complex febrile seizure (CFS). Ninety patients (17\%) had a previous simple febrile seizure. Of the total with CFS, $340(64 \%)$ had a lumbar puncture; $14(2.7 \%)$ had CSF pleocytosis. Three patients $(0.9 \%)$ had ABM; 2 had csf pleocytosis, diplococci, and a positive culture for Streptococcus pneumoniae. Of the 2 with confirmed ABM, 1 aged 4 years was found unresponsive at presentation and had posturing of one arm and anisocoria; the other aged 11 months was sleepy and on examination, appeared flaccid, toxic, with bulging fontanel, nuchal rigidity and apnea. The third child had failed the LP test (no csf cell count), she appeared well at presentation, but her blood culture grew $S$ pneumoniae and she was treated as suspected ABM. None of the patients discharged without LP returned with a diagnosis of ABM. The decision to perform LP with a first CFS should be based on clinical suspicion and additional signs and symptoms suggestive of meningitis. ABM in a child presenting with CFS is uncommon $(<1 \%)$, and the risk is particularly low with CFSs manifested by 2 brief nonfocal seizures in 24 hours. (Kimia A, Ben-Joseph EP, Rudloe T, et al. Yield of lumbar puncture among children who present with their first complex febrile seizure. Pediatrics July 2010;126:62-69). (Respond:Amir Kimia MD, Division of Emergency Medicine, Children's Hospital Boston, 300 Longwood Ave, Boston, MA 02115. E-mail: amir.kimia@childrens.harvard.edu).

COMMENT. The rate of seizure among children with acute bacterial meningitis is reported at $12 \%$ (Rosman NP et al. 1985) to $27 \%$ (Rosenberg NM et al. 1992). In the above series of patients diagnosed with a first complex febrile seizure, the rate of bacterial meningitis among those having an LP was quoted at $0.9 \%(0.6 \%$ in the total group). Routine LP is obviously inappropriate in all children with a complex febrile seizure, especially in those with short nonfocal seizures repeated in 24 hours. The 2 patients with confirmed ABM $(0.5 \%)$ in the Boston series had symptoms and signs of meningitis other than seizure. Furthermore, the description of presenting features was not convincing for a diagnosis of seizure.

Investigation of infections causing febrile seizures. In a study of 100 consecutive febrile seizures, LP was performed in 14 patients ( 11 complex seizures and 3 simple). Clinical suspicion and complex seizures were the principal indications for LP, and not age. Viral infection was the most common cause of fever, and bacterial infection was infrequent. Bacterial cultures performed in all 100 patients were positive in only $5 \%$, none from cerebrospinal fluid. Rapid viral testing and diagnosis would result in less emphasis on need for LP and a reduction in empiric antibacterial treatment. Complex 
febrile seizure as an indication for lumbar puncture should be reexamined. Patients who recover consciousness rapidly should be observed, and the tap deferred. (Millichap JJ et al. Methods of investigation and management of infections causing febrile seizures. Presented at AAN 60 ${ }^{\text {th }}$ Annual Meeting, 2008; Pediatr Neurol 2008;39:381-386). In this study, $23 \%$ of febrile seizures were complex, and of these $50 \%$ received LP, a slightly lower percentage than that reported in the Boston study.

\section{SPEECH AND LANGUAGE DISORDERS}

\section{FUNCTIONAL MRI NEURAL ACTIVATION PATTERNS IN EARLY AND LATE TALKERS}

Neural activation patterns, produced while listening to and reading words and non-words, were evaluated by functional MRI in 48 school children, mean age 8 years, in a study at Yale School of Medicine, New Haven, CT. Comparison of subsets of early, ontime and late talkers, matched for age, gender and performance IQ, showed that activation in bilateral thalamus and putamen, and left insula and superior temporal gyrus (circuits underlying speech and language) during these tasks was significantly lower in late talkers. Age of language acquisition has effects on reading and language behavior, and on corresponding cortical and subcortical neural circuitry. Late talkers were at risk for reading problems. The findings demonstrate the importance of early language development on formation of critical neural circuits and the need for prompt identification of language delays. (Preston JL, Frost SJ, Mencl WE, et al. Early and late talkers: school-age language, literacy and neurolinguistic differences. Brain August 2010;133:2185-2195). (Respond: Jonathan Preston, Haskins Laboratories, 300 George St, Suite 900, New Haven, CT 06511. E-mail: preston@haskins.yale.edu).

COMMENT. This study underscores previous findings of the effect of age of talking on the development of language and literacy. Talking is strongly related to neural activation patterns, particularly in subcortical regions, putamen and thalamus, regions implicated in the Galaburda studies of dyslexia.

\section{LEFT OCCIPITO-TEMPORAL LOCALIZATION OF READING EPILEPSY}

Researchers at Hopital de la Timone, Marseille, France, report a 28-year-old woman with a history of a febrile seizure at age 4 years and recent onset of partial seizures precipitated by silent reading. Left occipito-temporal localization of the seizures was confirmed by EEG, PET and SPECT. During a seizure, she was unable to understand what she was reading, and after looking up from the page, she saw letters and words, had a feeling of strangeness, right hemi-body jerks, and secondary seizure generalization. Seizures were controlled with carbamazepine. Interictal EEG showed left temporal posterior spikes, and during video-EEG a seizure was recorded $5 \mathrm{~min}$ after silent reading of a woman's magazine, with left occipito-temporal predominance. Interictal PET showed bilateral occipito-temporal hypometabolism with left predominance, and ictal 